\title{
Composición, riqueza de especies y abundancia de insectos defoliadores de actividad nocturna asociados a Aristotelia chilensis (maqui) en el bosque maulino fragmentado
}

\author{
Composition, species richness and abundance of nocturnal folivorous insects associated \\ with Aristotelia chilensis (maqui) in the fragmented Maulino forest
}

XAVIERA DE LA VEGA ${ }^{1} \&$ AUDREY A. GREZ ${ }^{2 *}$

\author{
${ }^{1}$ Departamento de Ciencias Ecológicas, Facultad de Ciencias, Universidad de Chile. 16 Casilla 653, Santiago, Chile \\ ${ }^{2}$ Departamento de Ciencias Biológicas Animales, Facultad Ciencias Veterinarias y Pecuarias, Universidad de Chile, Casilla \\ 2, Correo 15, La Granja, Santiago, Chile; \\ *e-mail para correspondencia: agrez@uchile.cl
}

\begin{abstract}
RESUMEN
En el bosque maulino, la herbivoría sobre Aristotelia chilensis (maqui) es negativamente afectada por la fragmentación del bosque, siendo mayor en el bosque continuo que en los fragmentos, particularmente a inicios de la temporada de crecimiento. Este fenómeno puede deberse a cambios en la dinámica de las poblaciones de defoliadores, esencialmente insectos. En este trabajo se evaluó la abundancia, riqueza de especies y composición de insectos defoliadores de actividad nocturna presentes en A. chilensis en un bosque continuo (600 ha) y en ocho fragmentos remanentes (0,4-20 ha). Los muestreos se realizaron mensualmente, entre agosto de 2005 y febrero de 2006, en 32 ejemplares adultos de A. chilensis en el bosque continuo y en 32 ejemplares en los fragmentos. Los insectos fueron muestreados durante las cinco primeras horas de la noche. Se recolectaron 890 insectos defoliadores, pertenecientes a 17 familias y 77 especies pertenecientes a los órdenes Coleoptera, Orthoptera y Lepidoptera, siendo todas nativas. La abundancia total no varió según el hábitat. Sin embargo, la fragmentación incrementó o disminuyó la abundancia de algunas especies. La riqueza de especies por árbol tampoco fue afectada por la fragmentación del bosque, aunque el número total de especies fue considerablemente mayor en los fragmentos que en el bosque continuo. La similitud de especies fue mayor dentro del bosque continuo que entre el bosque continuo y los fragmentos o que entre los fragmentos. A principios de la temporada de crecimiento de A. chilensis (septiembre), la abundancia de Sericoides obesa fue significativamente mayor en el bosque continuo que en los fragmentos. Al avanzar en la temporada, Sericoides viridis se hizo más abundante en los fragmentos. Por el tamaño y la voracidad de los insectos del género Sericoides ellos serían los principales responsables de los patrones de defoliación de $A$. chilensis en el bosque maulino.
\end{abstract}

Palabras clave: insectos defoliadores, bosque maulino, fragmentación del hábitat.

\begin{abstract}
At the Maulino forest, herbivory upon Aristotelia chilensis (maqui) is negatively affected by forest fragmentation, being higher in the continuous forest than in the small remnant fragments, particularly at the beginning of the growing season (spring). This phenomenon may be attributable to changes in the population dynamics of herbivores, mostly insects. In this work, the effect of the Maulino forest fragmentation on the abundance, species richness and composition of nocturnal defoliating insects associated with A. chilensis was evaluated. The insects associated with A. chilensis were sampled monthly, between August 2005 and February 2006, in 32 trees located in a continuous forest (600 ha) and in 32 trees located in eight forest fragments (0.4-20 ha). Insect sampling was carried out during the first five night hours. 890 folivorous insects from 17 families and 77 species of Coleoptera, Orthoptera and Lepidoptera were collected. All species were native. The total abundance did not vary with habitat fragmentation. Nevertheless, some species increased their abundance with fragmentation, while others were depressed. The species richness of folivorous insects per tree did not vary with forest fragmentation, though the total number of species was considerably higher in the forest fragments. The species similarity was higher within the continuous forest than between the continuous forest and fragments or among fragments. At the beginning of the season (September), the abundance of Sericoides obesa was significantly higher in the continuous forest than in the forest fragments. Later in the season, Sericoides viridis became more abundant in the forest fragments. Because of the body size and the voracity of Sericoides sp., these insects seem to be responsible of the herbivory patterns of A. chilensis at the Maulino forest.
\end{abstract}

Key words: folivorous insects, Maulino forest, habitat fragmentation. 


\section{INTRODUCCIÓN}

La deforestación y fragmentación de los bosques son fenómenos que ocurren a escala global y constituyen problemas ambientales apremiantes por su impacto sobre la biodiversidad (Myers 1988, Groom \& Schumaker 1993, FAO 2002). Los cambios en la identidad, diversidad o abundancia poblacional de las especies, producto de la fragmentación, pueden alterar las interacciones ecológicas y los procesos ecosistémicos, componentes funcionales de la biodiversidad (sensu Noss 1990). Entre las interacciones ecológicas afectadas por la fragmentación del hábitat están las interacciones planta-animal, como la herbivoría. Cambios en esta interacción son relevantes por cuanto la pérdida de área foliar puede tener consecuencias en el desempeño de las plantas, ya sea disminuyendo la producción de frutos (Crawley 1985) o aumentando el aborto de frutos (Hochwender et al. 2003), conllevando finalmente a cambios en la estructura del bosque (Ricklefs \& Miller 1999). Los escasos antecedentes de la literatura sugieren que, en general, la herbivoría disminuye con la fragmentación (e.g., Roland 1993, Benítez-Malvido et al. 1999, Bresciano et al. 1999, Benítez-Malvido 2001, Arnold \& Asquith 2002, Vásquez et al. 2007). Por ejemplo, en bosques tropicales experimentalmente fragmentados en Manaos, la herbivoría sobre plántulas al inicio de la temporada, es más baja en fragmentos de bosque que en un bosque continuo, aunque pasado algunos años tal diferencia desaparece (Benítez-Malvido et al. 1999). Esta disminución en la herbivoría se debería a efectos depresores de la fragmentación sobre la abundancia o riqueza de especies de herbívoros (Benítez-Malvido et al. 1999).

El bosque maulino, ubicado en la cordillera de la Costa de la Región del Maule, Chile, entre $\operatorname{los} 35^{\circ} 55^{\prime}$ y $37^{\circ} 20^{\prime} \mathrm{S}$, ha sido intensamente deforestado y fragmentado (Grez et al. 1997) con una reducción del área del bosque original de un $67 \%$ entre los años 1975 y 2000, quedando hoy en día distribuido en pequeños fragmentos rodeados por plantaciones de Pinus radiata (Grez et al. 1997, Bustamante \& Castor 1998, Echeverría et al. 2006). Este bosque es de gran interés biológico debido al alto endemismo de su biota y por ser un centro de confluencia de la biota austral y de asociaciones más nortinas como el bosque esclerófilo (Saavedra \& Simonetti 2001), siendo considerado un punto caliente para la conservación de biodiversidad (Myers et al. 2000).

La fragmentación ha provocado importantes cambios en la biota de este bosque. Así, los carnívoros y algunas familias de insectos disminuyen su abundancia en los pequeños fragmentos de bosque, en cambio, los coleópteros epígeos, las aves insectívoras, los mamíferos pequeños y el pudú son más abundantes en estos remanentes en comparación con un área extensa (600 ha) de bosque continuo (véase Bustamante et al. 2006 para una revisión). Estos cambios en las abundancias poblacionales han implicado alteraciones en la intensidad de algunas interacciones ecológicas, como la granivoría, insectivoría y herbivoría (Simonetti et al. 2006).

En el bosque maulino, la herbivoría de diferentes especies de plantas, tanto en su estado adulto como juvenil, disminuye en los fragmentos remanentes (Vega 2001, Vásquez et al. 2007, Simonetti et al. en prensa). En particular, la remoción de superficie foliar en Aristotelia chilensis (Mol.) Stuntz (Elaeocarpaceae), el maqui, es significativamente menor en los pequeños fragmentos remanentes que en el bosque continuo, particularmente a inicios de la temporada de crecimiento de nuevas hojas. Luego estas diferencias comienzan a desaparecer, siendo al final de la temporada la herbivoría similar en fragmentos de bosque y bosque continuo (Vásquez et al. 2007). Este fenómeno puede deberse a cambios en la dinámica de las poblaciones de defoliadores, esencialmente insectos, debido a que estos son los herbívoros más importantes en este bosque (Vega 2001). Sin embargo, esta hipótesis aún no ha sido puesta a prueba. Observaciones preliminares en maqui sugieren que los insectos herbívoros asociados a esta planta, como Sericoides sp. (Coleoptera: Scarabaeidae), tienen una actividad defoliadora principalmente en la noche (Artigas 1994) y que los insectos herbívoros diurnos son escasos (Jaña-Prado \& Grez 2004). Por ello, un muestreo nocturno es necesario para evaluar los posibles efectos de la 
fragmentación sobre este gremio de insectos. El objetivo de este trabajo fue evaluar si la fragmentación del bosque maulino afecta la abundancia, riqueza y composición de insectos defoliadores de actividad nocturna presentes en A. chilensis y si estas variaciones se relacionan con los patrones de herbivoría descritos por Vásquez et al. (2007).

\section{MATERIALES Y MÉTODOS}

\section{Lugar del estudio}

El estudio se realizó en la Reserva Nacional Los Queules (RNLQ) y en ocho fragmentos remanentes de bosque maulino vecinos a la reserva, pero separados de ella y entre sí por al menos $50 \mathrm{~m}$. La RNLQ (35\%41'19" S, $72^{\circ} 41^{\prime} 15^{\prime \prime}$ O) se encuentra ubicada a $71 \mathrm{~km}$ al oeste de Cauquenes, en el sector de Tregualemu que, junto a terrenos vecinos de propiedad privada, suman un área de 600 ha de bosque maulino, una de las áreas más extensas de este bosque existente hoy en día. Los fragmentos remanentes de bosque comprenden áreas menores a 20 ha, y se encuentran aislados entre sí y de la reserva por plantaciones de pino (para un mapa del área véase Donoso et al. 2004). Las especies dominantes son el hualo (Nothofagus glauca) y el roble (Nothofagus obliqua) que coexisten con especies endémicas que se encuentran en peligro de extinción como el queule (Gomortega keule), ruil (Nothofagus alessandrii) y pitao (Pitavia punctata) (San Martín \& Donoso 1996). En el bosque maulino A. chilensis es una especie abundante, presente tanto en los fragmentos de bosque como en el bosque continuo y en la matriz de pino, pudiendo en el futuro relegar a las especies que dominan actualmente en los fragmentos (hualo y roble) (Bustamante et al. 2005). De hecho, ya hoy la densidad de este árbol es significativamente mayor en los fragmentos que en el bosque continuo (media \pm error estándar $=43,9 \pm 6,18$ y 4,2 $\pm 0,99$ árboles por $400 \mathrm{~m}^{2}$, respectivamente) (Lagos 2007).

\section{Diseño experimental y muestreo de insectos}

Los insectos defoliadores (i.e., Coleoptera, Lepidoptera y Orthoptera) asociados a $A$. chilensis fueron muestreados mensualmente desde agosto de 2005 a febrero de 2006, época de mayor actividad de insectos en Chile central y en el bosque maulino (Fuentes et al. 1981, Jaña-Prado \& Grez 2004) y que coincide con el periodo de crecimiento vegetativo de $A$. chilensis en la que se concentra su defoliación (Vásquez et al. 2007). Tanto en el bosque continuo como en los 8 fragmentos de bosque se seleccionaron aleatoriamente 32 ejemplares adultos de A. chilensis (total =64). En los fragmentos y en el bosque continuo los árboles seleccionados se hallaban separados por al menos $10 \mathrm{~m}$ de distancia y en el caso del bosque continuo, ellos se distribuyeron a lo largo de un transecto de $700 \mathrm{~m}$ de largo, aproximadamente.

Los insectos fueron muestreados durante las cinco primeras horas después del anochecer, a lo largo de tres noches de cada mes, bajo condiciones climáticas similares. Cada noche, se incluyeron en el muestreo tanto árboles de los fragmentos como del bosque continuo. En cada ejemplar escogido se seleccionaron al azar cinco ramas, cuyas hojas fueron observadas por el haz y el envés durante aproximadamente $10 \mathrm{~min}$ por árbol, recolectando los insectos presentes. Este periodo de tiempo resultó ser adecuado, incluso superior al necesario para evaluar la fauna presente en cinco ramas, por cuanto antes de este lapso ya no se observan insectos adicionales. Posterior a estas observaciones se utilizó el método de sacudido de follaje sobre paraguas entomológico, sacudiendo dos veces todo el follaje de cada árbol y colectando los insectos en un paño blanco de $1 \mathrm{~m}^{2}$ dispuesto bajo las ramas.

Las larvas de lepidópteros fueron llevadas a una cámara de crianza, suministrándoles hojas de maqui como alimento hasta que alcanzaran el estado adulto y así poder identificarlas. En tanto, los coleópteros y los ortópteros fueron almacenados en tubos Eppendorf con alcohol de $70^{\circ}$. Los insectos fueron identificados a nivel de morfoespecie o especie cuando fue posible, a través de claves taxonómicas y comparaciones con colecciones entomológicas del Museo Nacional de Historia Natural y del Museo Entomológico Luis Peña de la Facultad de Ciencias Agronómicas de la Universidad de Chile. Los insectos fueron considerados defoliadores según recomendaciones de especialistas. 


\section{Análisis estadístico}

Debido a que no existe en el área otra unidad de bosque continuo de estas dimensiones, las muestras de este tipo de bosque fueron tomadas de la misma unidad experimental, lo que podría significar que estas muestras no hubiesen sido estadísticamente independientes. Por ello, para probar la independencia espacial de las muestras, y usarlas como réplicas en los análisis estadísticos, se aplicó la prueba de Mantel (Fortin \& Gurevitch 1993). Esta prueba demostró que las diferencias en abundancia y riqueza de insectos herbívoros entre árboles dentro del bosque continuo y dentro de los fragmentos fueron espacialmente independientes (abundancia, $\mathrm{r}_{\mathrm{s}}=-0,13 ; \mathrm{P}=0,76$ para bosque continuo y $\mathrm{r}_{\mathrm{S}}=-0,13 ; \mathrm{P}=0,77$ para fragmentos; riqueza, $r_{s}=0,01 ; \mathrm{P}=0,74$ para bosque continuo y $\mathrm{r}_{\mathrm{s}}=0,02 ; \mathrm{P}=0,56$ para fragmentos). Por ello, cada árbol fue considerado como réplica.

La abundancia poblacional (número de individuos por árbol) y la riqueza de especies (número de especies por árbol) de insectos defoliadores en bosque continuo y fragmentos se comparó mediante un análisis de varianza para medidas repetidas (ANDEVAmr), con las fechas de muestreo como medida repetida, usando el programa computacional Statistica 6.0 (Statsoft Inc. 2001). Los datos fueron transformados con $\log _{10}(x+1)$, para cumplir con el supuesto de normalidad. Como prueba a posteriori se utilizó la prueba de Tukey con a $=0,05$.

La similitud en la composición de insectos herbívoros entre el bosque continuo y los fragmentos (considerando las capturas acumuladas durante todo el estudio) se estimó mediante el coeficiente de Sorensen (Krebs 1989) y el índice de Morisita-Horn (Moreno 2001). Estos índices se calcularon de dos maneras: primero comparando la totalidad de especies encontradas en todos los árboles muestreados en el bosque continuo y en los ocho fragmentos $\mathrm{y}$, segundo, agrupando los datos en ocho bloques para bosque continuo y ocho para fragmentos, de manera de tener una variabilidad al interior del bosque continuo. Cada bloque estuvo compuesto por cuatro árboles, los que en el bosque continuo correspondieron a los cuatro árboles más cercanos dentro del transecto y en los fragmentos a los cuatro árboles muestreados dentro de cada fragmento.
Adicionalmente, para representar gráficamente la similitud de especies entre los bloques del bosque continuo y de los fragmentos, se construyó un dendrograma a través de un análisis de conglomerados, el que consideró las abundancias de todas las especies, excluyendo a Lepidoptera sp., por cuanto gran parte de este grupo no fue identificado a nivel de especie (i.e., lepidópteros criados y que no llegaron a adultos). El análisis se realizó mediante el método de agrupamiento Encadenamiento promedio o UPGMA, usando el programa InfoStat (InfoStat 2006). En el análisis, la distancia utilizada fue la euclidiana. La significancia estadística de las agrupaciones observadas se determinó a partir de una prueba de aleatorización, utilizando el programa PopTools 2.7.5 (Poptools 2006).

\section{RESULTADOS}

A lo largo del estudio se colectaron 890 individuos de insectos defoliadores pertenecientes a Coleoptera, Orthoptera y Lepidoptera, de 17 familias y 77 especies (Tabla 1). El 93,5 \% de las especies fueron identificadas a nivel de especie o morfoespecie, siendo todas nativas.

En general Coleoptera fue el orden con mayor abundancia, representando el 77,5\% del total de individuos capturados, seguido por Lepidoptera con $12,4 \%$ y Orthoptera con $10,1 \%$ del total de individuos. Las familias más comunes fueron: Chrysomelidae, Scarabaeidae y Elateridae, todas pertenecientes a Coleoptera.

Desde agosto a diciembre la familia más abundante fue Chrysomelidae, siendo en estos meses sus especies más representadas: Protopsilapha pallens (Blanchard), Protopsilapha pyrroptera (Phil \& Phil), Psathyrocerus sp. 1 y Psathyrocerus fulvipes Blanchard. En enero, en cambio, la familia con más individuos fue Scarabaeidae y la especie más habitual fue Sericoides viridis (Solier). En febrero, Chrysomelidae volvió a ser la familia más abundante pero $S$. viridis, al igual que en el mes anterior, fue la especie más abundante. Además de $P$. pallens, $P$. pyrroptera y $P$. fulvipes, durante todo el periodo de muestreo Deromecus pallipes (Solier) (Coleoptera: Elateridae) fue otra especie abundante. 


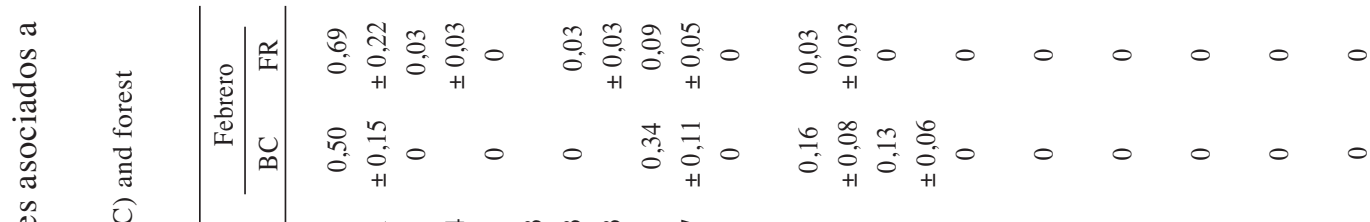

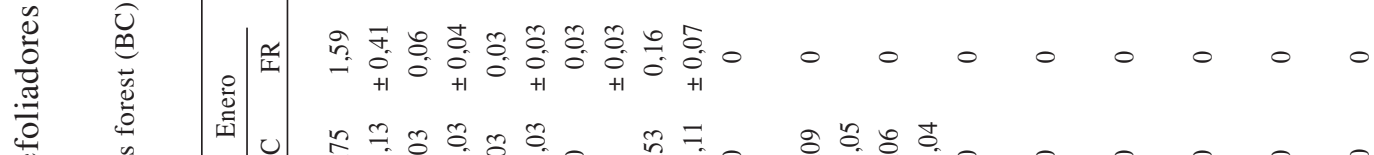

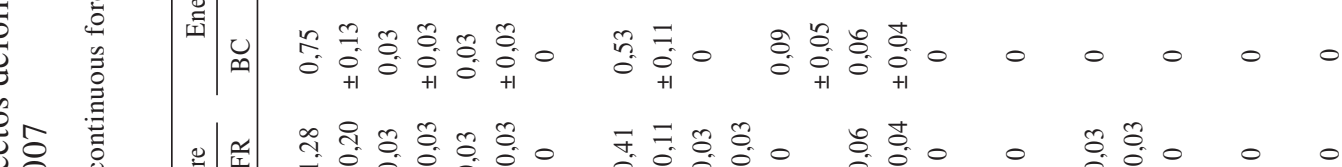

\section{这}

$\exists$

o.

空这

- $\rightarrow 2$

$+18$

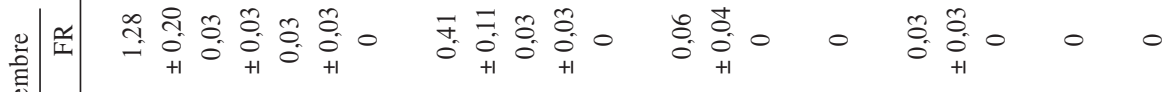

药|

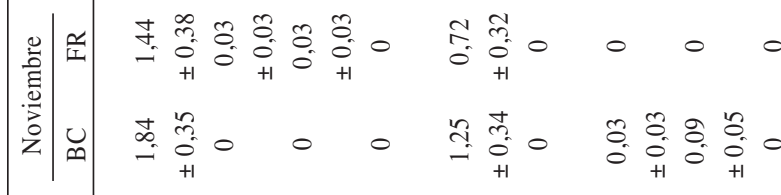

吾 艺

宅

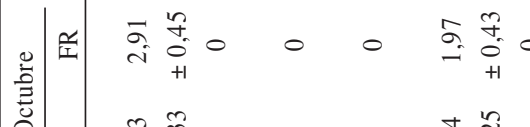

- 0

$\circ$

¿

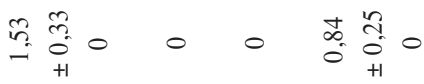

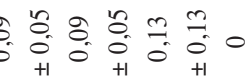

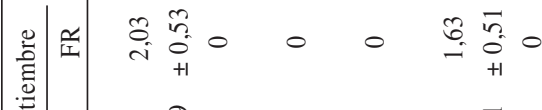

$\circ$

o

- 0 0 年主

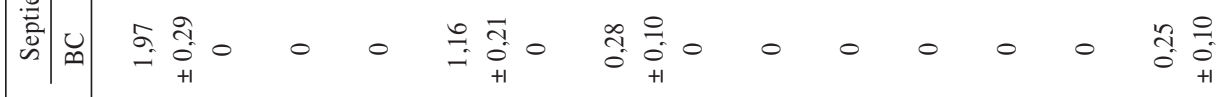

党离

至

可

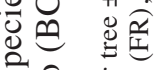

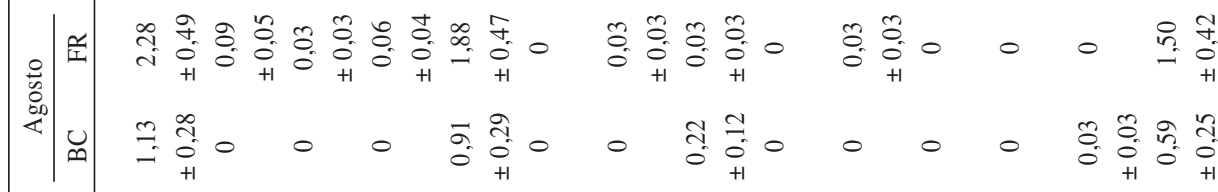

$\infty \quad+\quad$

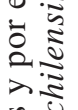




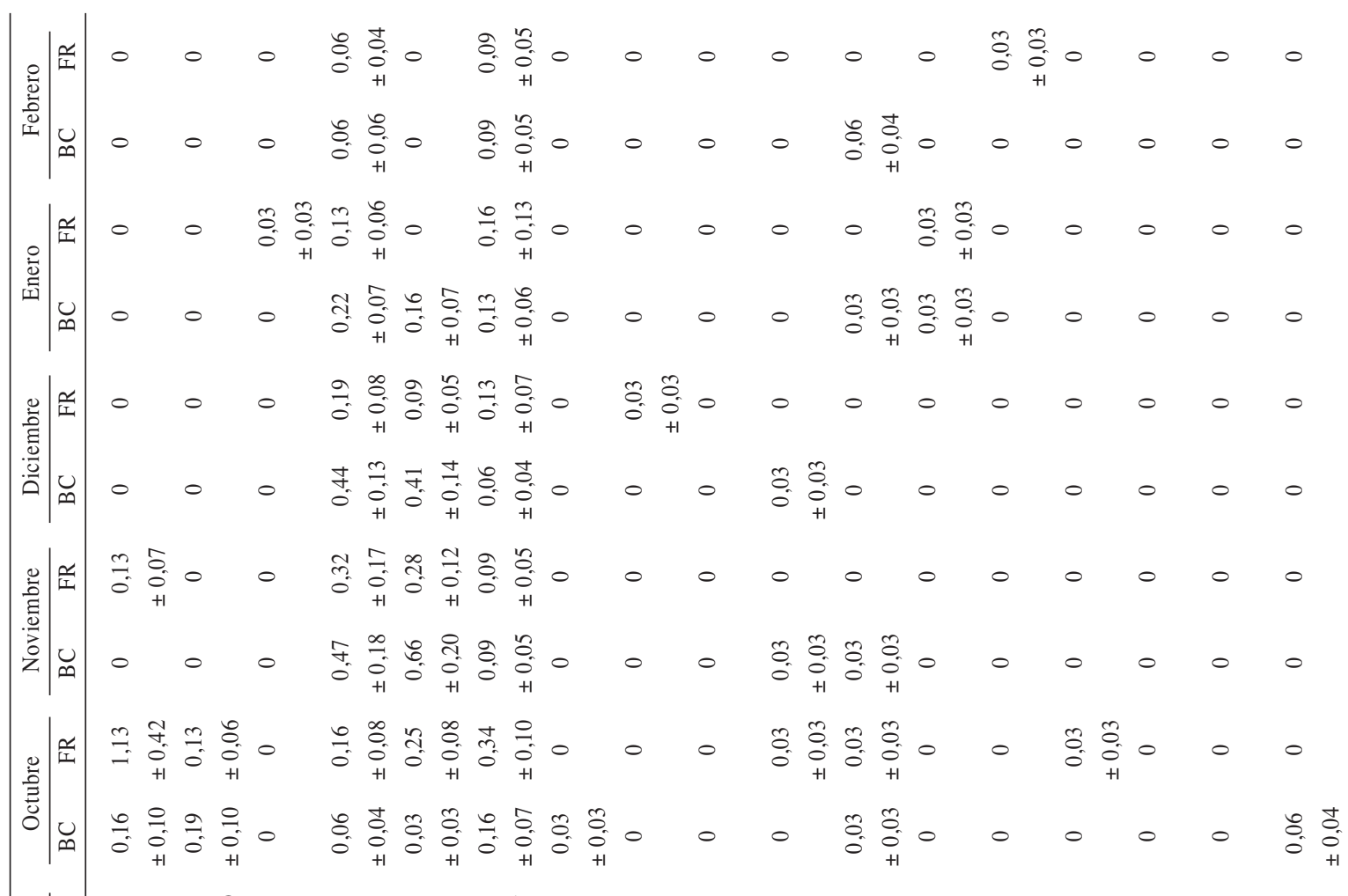

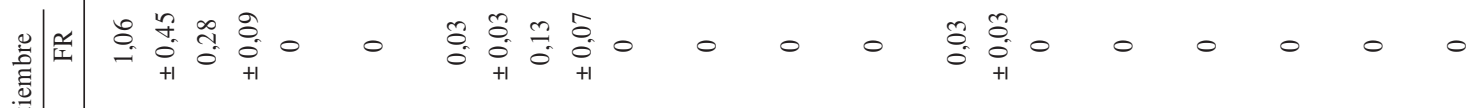

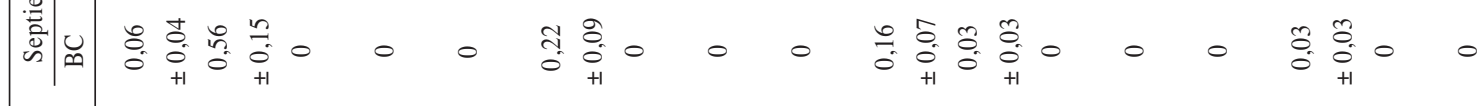
窟

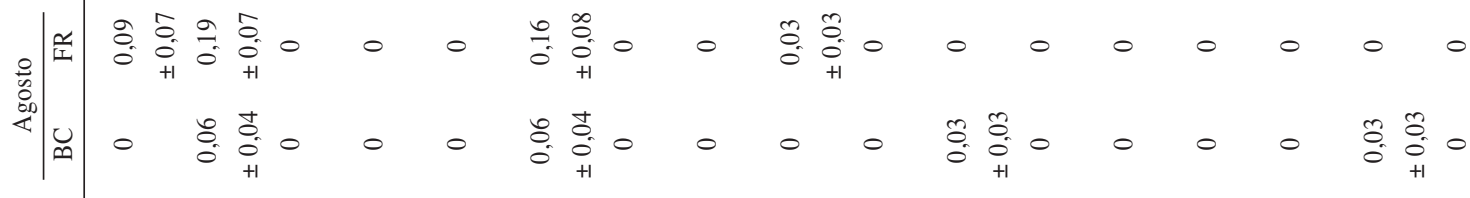
嵒尊

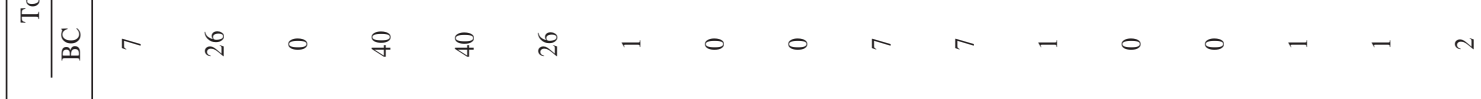

\section{言}




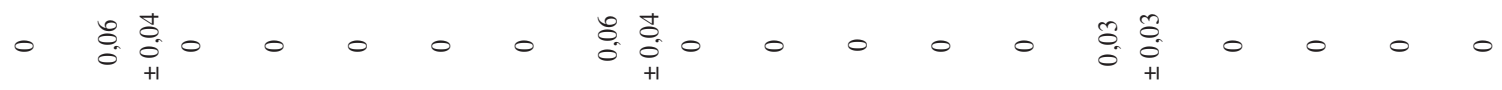

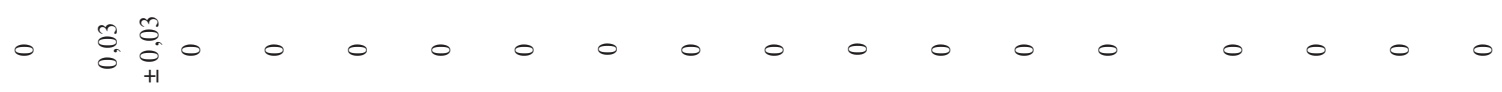

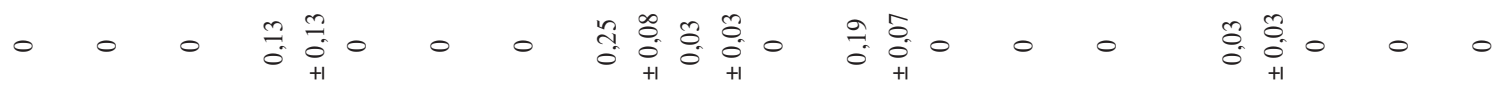

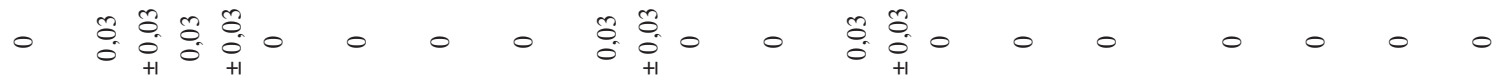

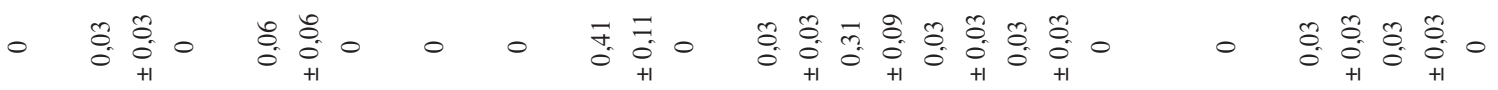

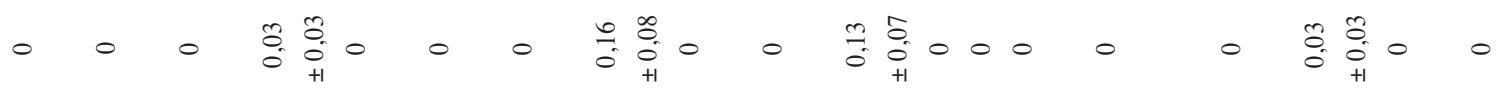

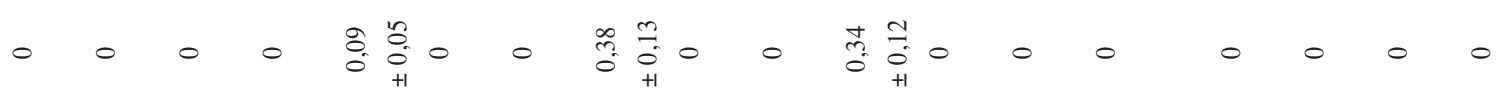

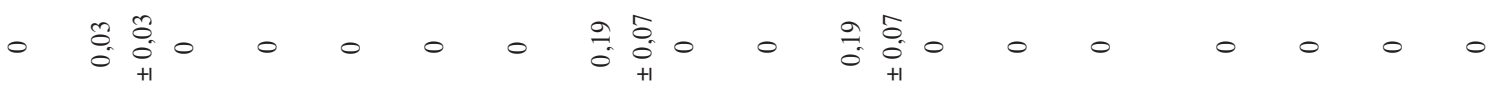

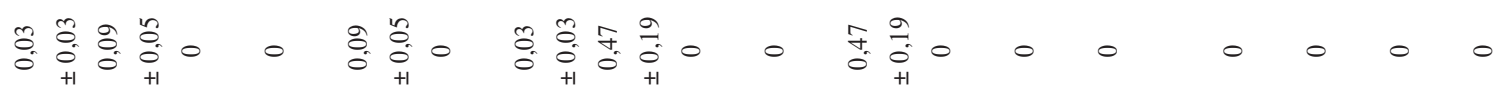

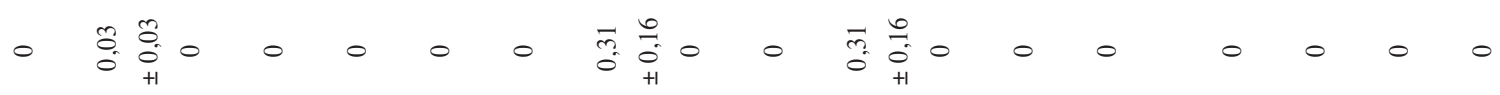

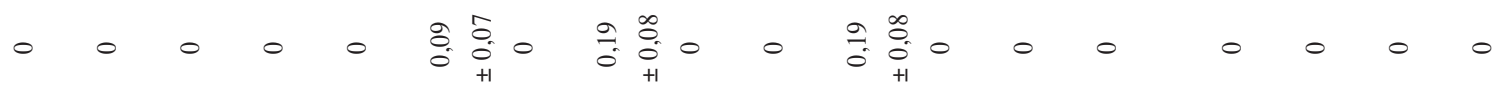

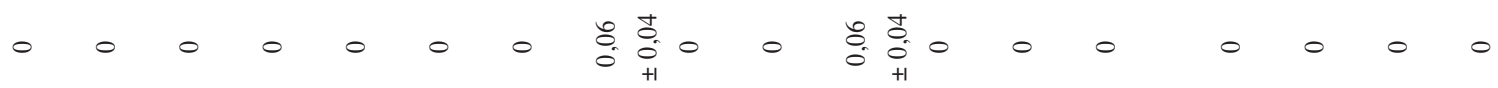

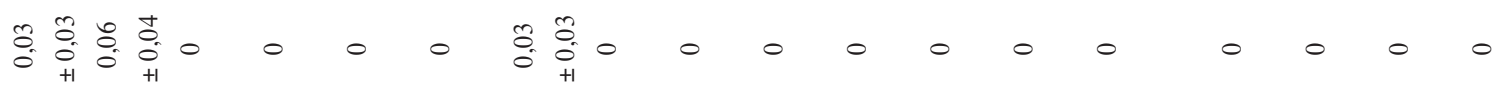

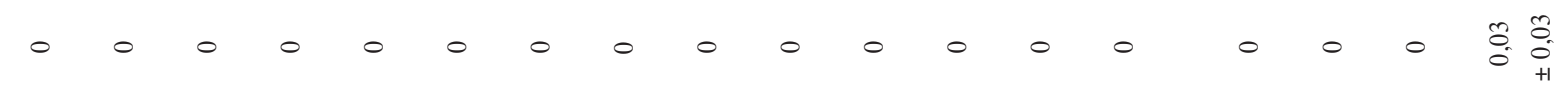

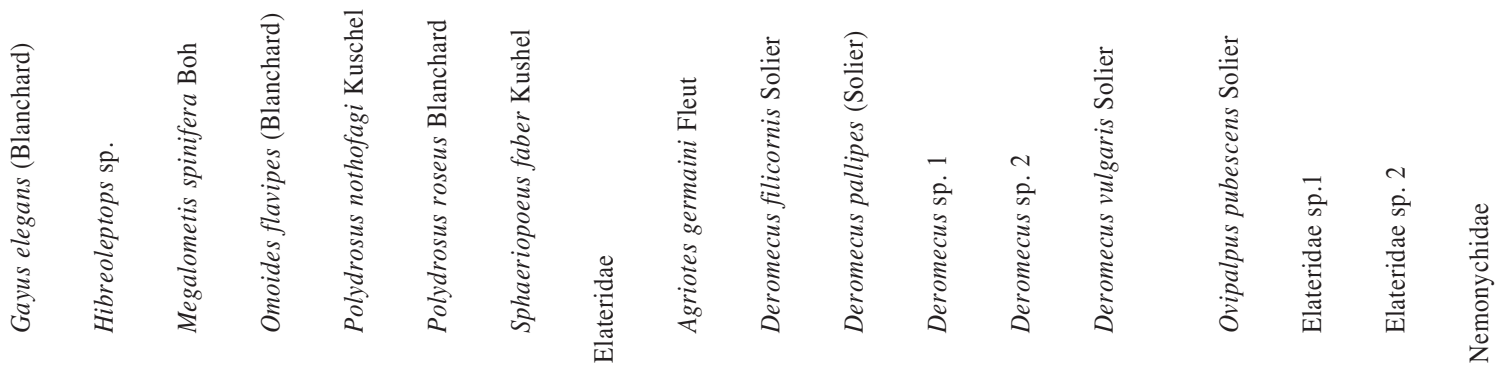




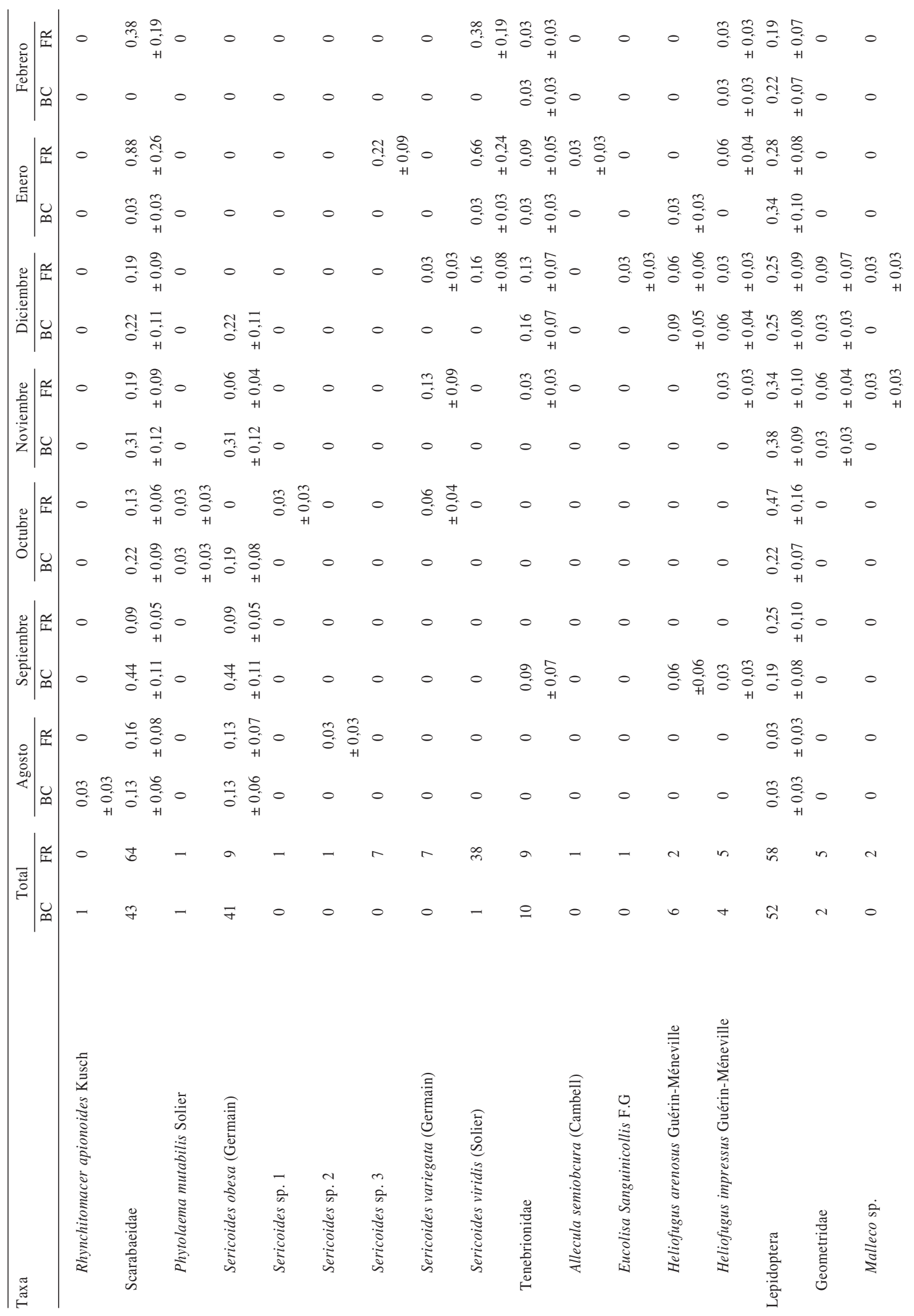




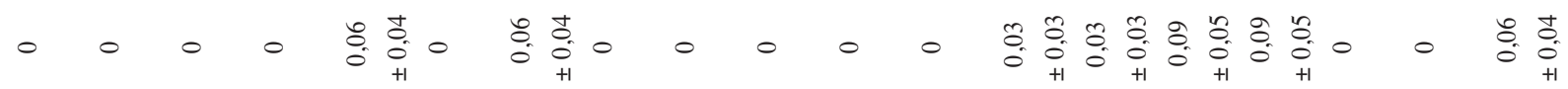

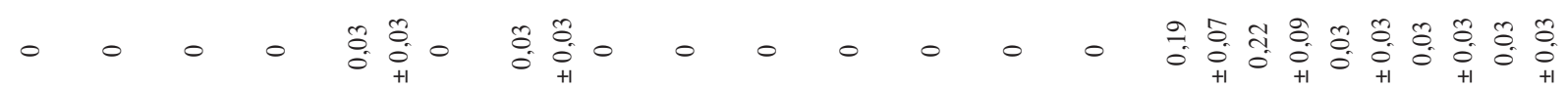

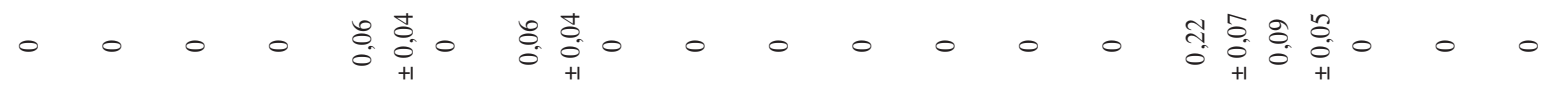

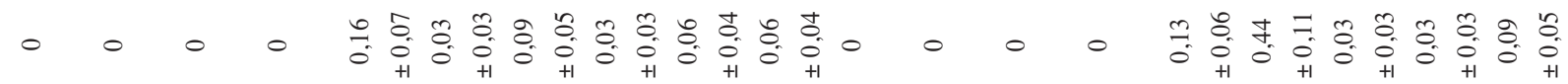
$\left.\begin{array}{llllllllllll}8 & 0 \\ 0 & 0 \\ 0 & 0\end{array}\right]$

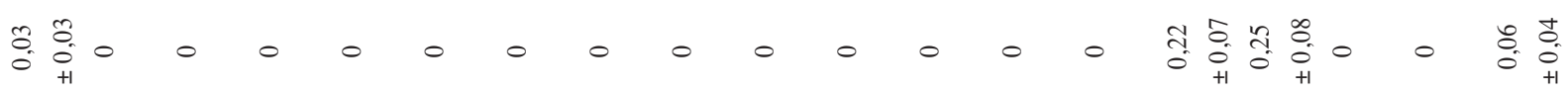

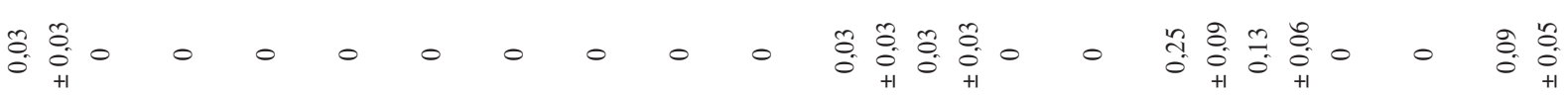

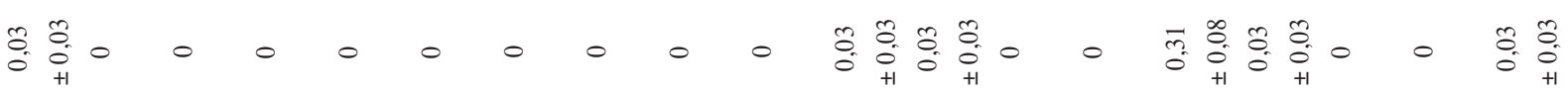

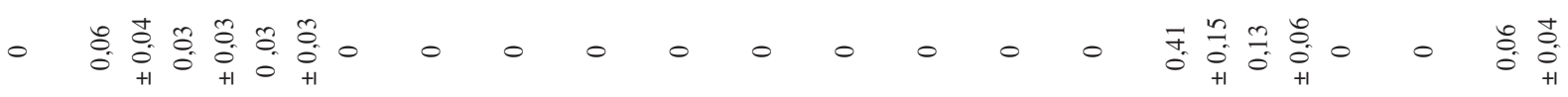

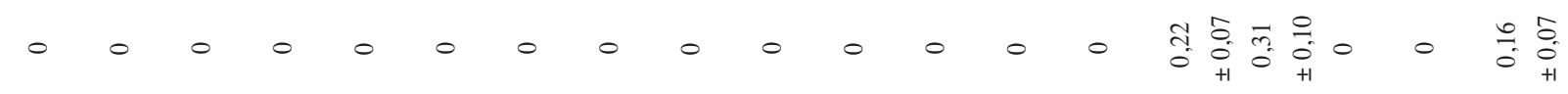

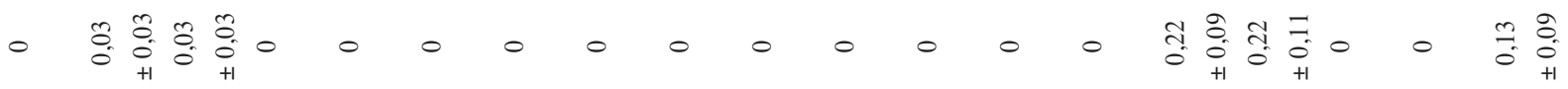

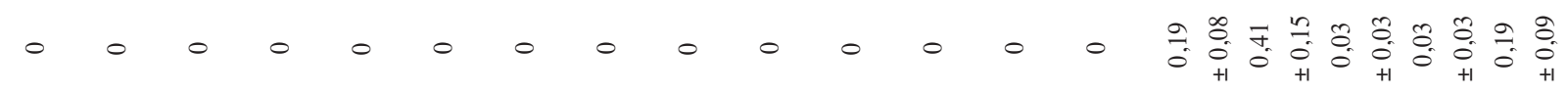

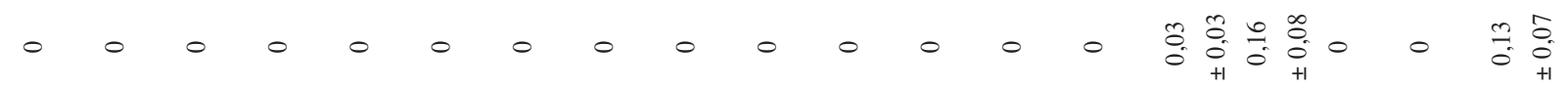

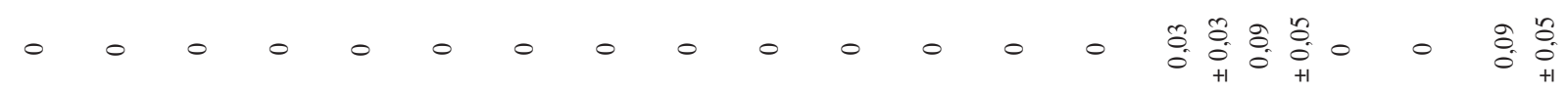

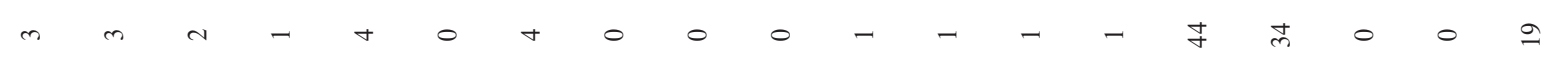

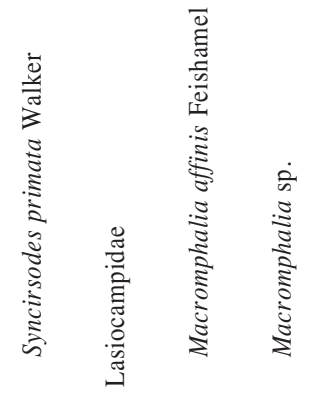

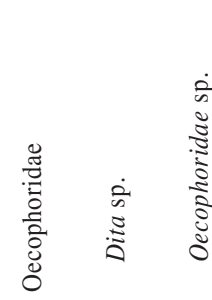

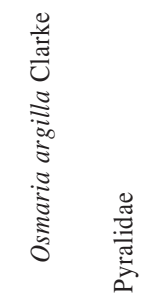

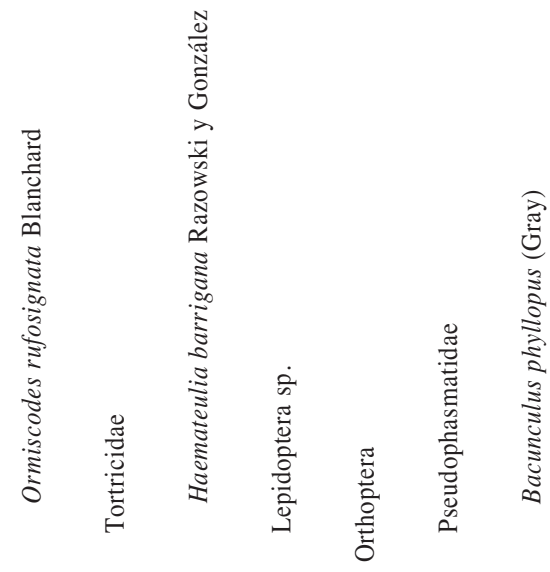




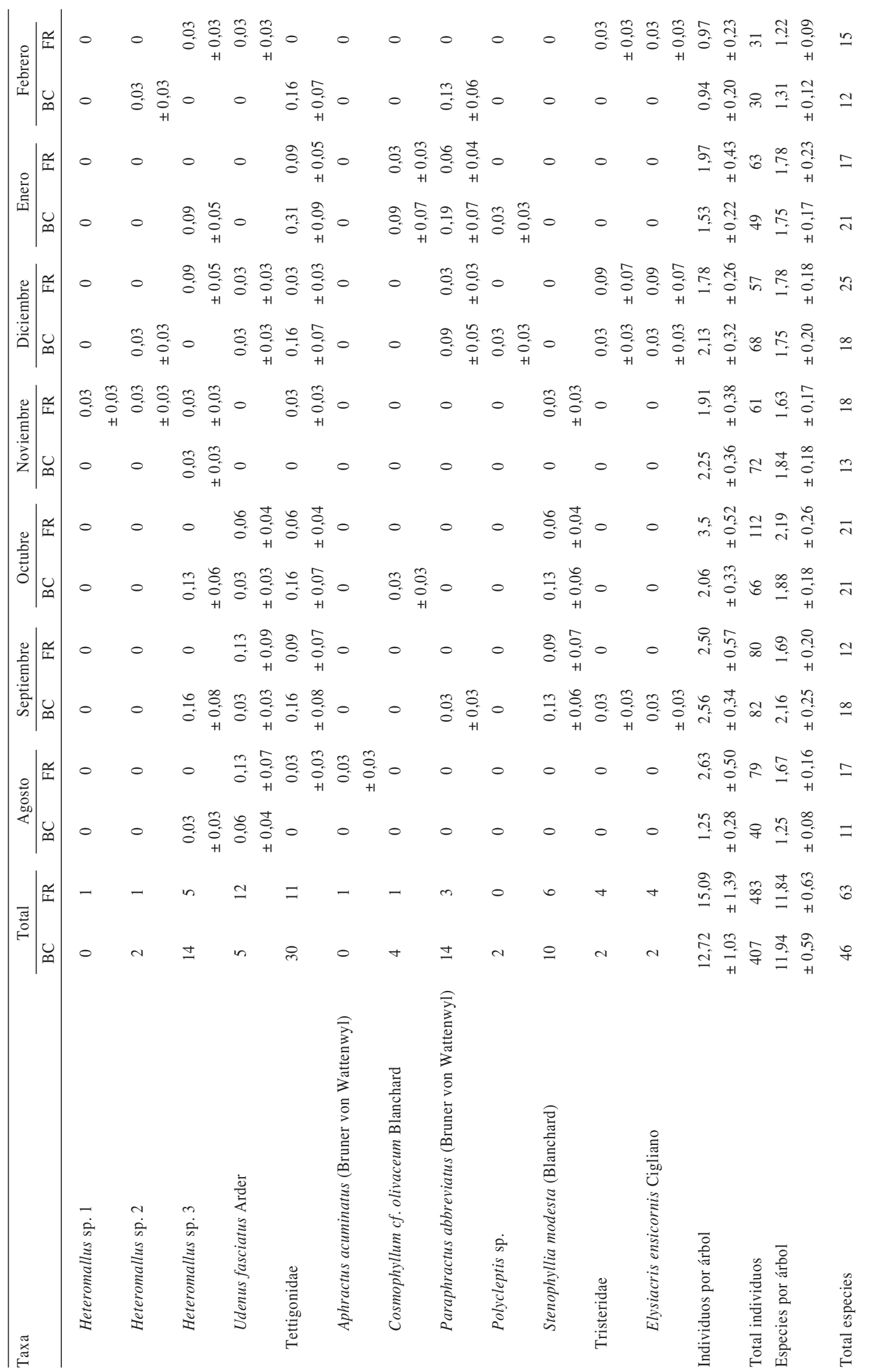


En cuanto al número de especies dentro de cada orden, de las 77 especies colectadas, Coleoptera acumula el $72,7 \%$ (56 especies), seguida por Orthoptera con un $14,3 \%$ (11) y por Lepidoptera con un $13 \%$ (10 especies) del total de especies. Las familias con mayor riqueza fueron, en Coleoptera, Curculionidae (18 especies), Chrysomelidae (14 especies) y Elateridae (nueve especies); en Lepidoptera, Oecophoridae (tres especies) y en Orthoptera, Tettigonidae (cinco especies, Tabla 1).

\section{Abundancia de insectos defoliadores asociados a A. chilensis en fragmentos y bosque continuo}

Considerando todas las fechas de muestreo, la abundancia total de insectos defoliadores asociados a $A$. chilensis no varió entre bosque continuo y fragmentos (ANDEVAmr, efecto hábitat, $\left.\mathrm{F}_{1,62}=0,30 ; \mathrm{P}=0,59\right)$. Si bien en agosto y octubre la abundancia total fue levemente superior en los fragmentos que en el bosque continuo, estas diferencias no fueron estadísticamente significativas (ANDEVAmr, efecto hábitat*fecha, $\left.\mathrm{F}_{6,372}=2,06 ; \mathrm{P}=0,06\right)$. Desde finales de primavera (noviembre) hasta finales de verano (febrero) la abundancia total de insectos defoliadores fue disminuyendo, manteniéndose similar entre los dos tipos hábitat (Fig. 1A). A nivel de especies, entre los Chrysomelidae la abundancia de $P$. pallens fue mayor en los fragmentos que en el bosque continuo (ANDEVAmr, efecto hábitat, $\mathrm{F}_{1,62}=$ $3,84 ; \mathrm{P}=0,05)$. Esta diferencia ocurrió particularmente en agosto, cuando se capturó la mayor cantidad de individuos, en tanto la abundancia en el bosque continuo siempre fue baja (Fig. 1B). Protopsilapha pyrroptera en el bosque continuo solo fue capturada en septiembre y octubre, en cambio, en los fragmentos estuvo presente de agosto a noviembre. La abundancia de esta especie fue afectada por el tipo de hábitat, siendo siempre más abundante en los fragmentos (ANDEVAmr, efecto hábitat, $\mathrm{F}_{1,62}=9,73 ; \mathrm{P}=0,003$; Fig. 1C). La abundancia de Psathyrocerus sp. 1 no fue afectada por el tipo de hábitat (ANDEVAmr, efecto hábitat, $\left.\mathrm{F}_{1,62}=2,31 ; \mathrm{P}=0,13\right)$. Sin embargo, en noviembre y diciembre esta morfoespecie fue significativamente más abundante en el bosque continuo que en los fragmentos (ANDEVAmr, efecto hábitat*fecha, $\mathrm{F}_{4,248}=$ 4,21; P = 0,003; Fig. 1D). La abundancia de $P$. fulvipes nunca fue afectada por el tipo de hábitat (ANDEVAmr, efecto hábitat, $\mathrm{F}_{1,62}=1,44 ; \mathrm{P}=$ 0,23 , Fig. 1E).

Entre los Elateridae, D. pallipes fue siempre más abundante en los fragmentos de bosque que en el bosque continuo (ANDEVAmr, efecto hábitat, $\mathrm{F}_{1,62}=4,30 ; \mathrm{P}=0,04 ;$ Fig. $\left.1 \mathrm{~F}\right)$, en tanto entre los Scarabaeidae, $S$. obesa fue siempre más abundante en el bosque continuo que en los fragmentos (ANDEVAmr, efecto hábitat, $\mathrm{F}_{1,62}=11,44 ; \mathrm{P}<0,001 ;$ Fig. $\left.1 \mathrm{G}\right)$. En el bosque continuo esta especie se capturó entre agosto y diciembre, en cambio, en los fragmentos se capturó entre agosto y noviembre, pero en muy bajas abundancias. Por otra parte, $S$. viridis fue significativamente más abundante en los fragmentos que en bosque continuo (ANDEVAmr, efecto hábitat, $\mathrm{F}_{1,62}=$ 9,14; $\mathrm{P}<0,001)$, particularmente entre diciembre y febrero. Solo un individuo de $S$. viridis fue observado en el bosque continuo, lo cual ocurrió en enero (Fig. 1H).

\section{Riqueza de especies y composición de insectos defoliadores asociados a A. chilensis en frag- mentos y bosque continuo}

La riqueza de especies de insectos defoliadores por árbol no fue afectada por la fragmentación del bosque maulino (ANDEVAmr, efecto hábitat, $\mathrm{F}_{1,62}=0,16 ; \mathrm{P}=0,69 ;$ Fig. 2). Sin embargo, en total se capturaron más especies en los fragmentos que en el bosque continuo, con 63 y 46 especies, respectivamente (Tabla 1 ).

Considerando todo el periodo de estudio, 14 especies fueron capturadas solo en el bosque continuo, 32 solo en los fragmentos y 30 fueron compartidas por ambos hábitat. La similitud en la composición de especies entre bosque continuo y fragmentos según el índice de Sorensen fue de 0,59 y según el índice de Morsita-Horn fue de 0,60. Al hacer el análisis por bloques (ocho del bosque continuo y ocho de los fragmentos), en promedio la similitud según el Índice de Sorensen fue 0,77 entre los bloques del bosque continuo y 0,73 entre los bloques de los fragmentos, en tanto entre los bloques del bosque continuo y de los fragmentos fue 0,74. Según el índice de Morisita-Horn, la similitud de los bloques al interior del bosque continuo fue 0,17 , de los bloques de los fragmentos fue $0,09 \mathrm{y}$ entre los bloques del bosque continuo y de los fragmentos fue 0,13 . 
(A)

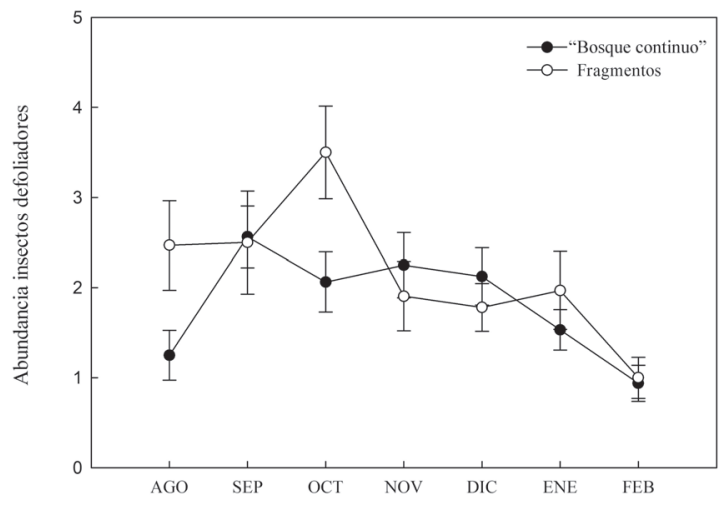

(C)

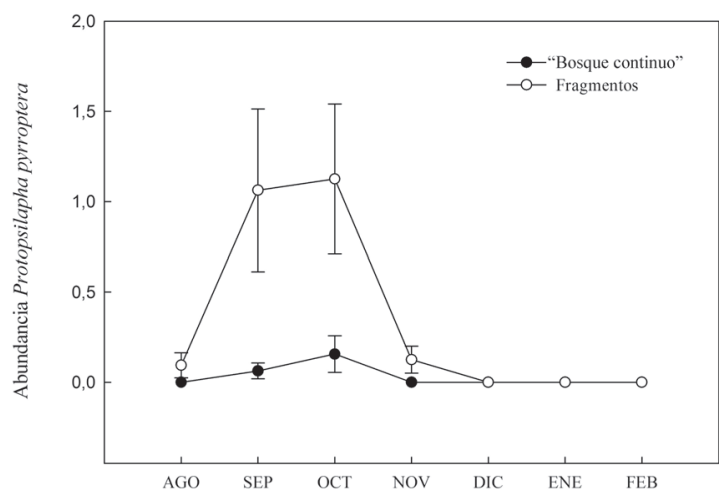

(B)

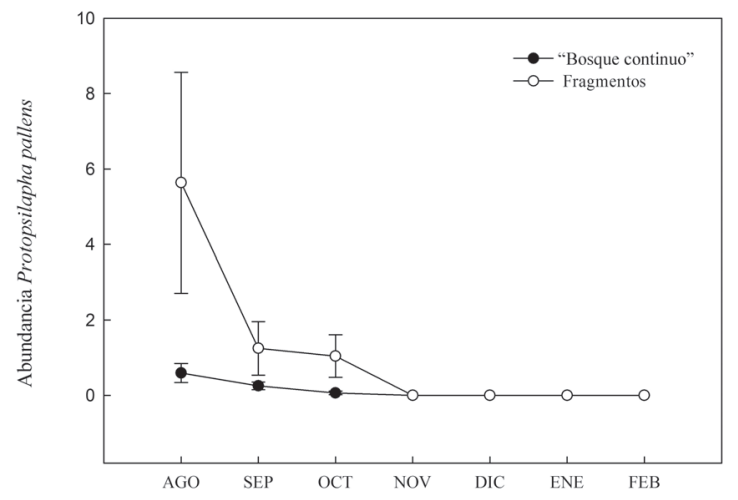

(D)

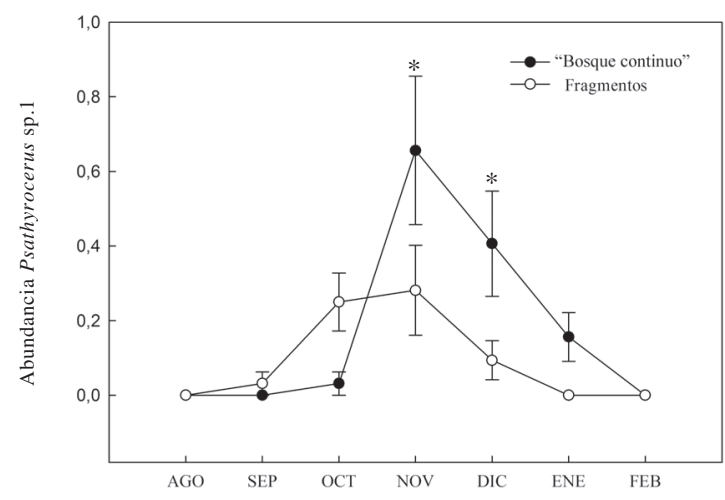

Fig. 1: (A) Abundancia total y abundancia de las especies más comunes; (B) Protopsilapha pallens, (C) Protopsilapha pyrroptera, (D) Psathyrocerus sp.1, (E) Psathyrocerus fulvipes, (F) Deromecus pallipes, (G) Sericoides obesa y (H) Sericoides viridis, de insectos defoliadores asociados a $A$. chilensis (promedio por árbol $\pm 1 \mathrm{EE}$ ) en el bosque continuo y fragmentos de bosque maulino, entre agosto 2005 y febrero 2006. El asterisco indica diferencias significativas (prueba de Tukey, $\mathrm{P}<$ $0,05)$.

(A) Total abundance and abundance of the commonest species; (B) Protopsilapha pallens, (C) Protopsilapha pyrroptera, (D) Psathyrocerus sp.1, (E) Psathyrocerus fulvipes, (F) Deromecus pallipes, (G) Sericoides obesa and (H) Sericoides viridis, of folivorous insects associated with A. chilensis (mean per tree \pm 1 SE) in the continuous forest and small fragments of Maulino forest, between August 2005 and February 2006. The asterisk indicates significant differences (Tukey test, $\mathrm{P}<0.05$ ).

El análisis de conglomerados muestra una mayor similitud entre los bloques del bosque continuo y entre los bloques de los fragmentos, aunque hubo excepciones en dos bloques de bosque continuo (BC6 y BC8) que fueron más similares a los fragmentos que a los otros bloques del mismo hábitat (Fig. 3). Además este análisis muestra que, en general, los bloques del bosque continuo son más similares entre sí que los bloques de los fragmentos (i.e., mayores distancias euclidianas entre los bloques de los fragmentos).

\section{DISCUSIÓN}

No se encontraron diferencias en la abundancia total de insectos defoliadores asociados a $A$. chilensis en el bosque continuo y los fragmentos de bosque maulino, lo cual en alguna medida pudo estar influenciado por la alta variabilidad de los datos, lo que podría haber disminuido la capacidad de las pruebas estadísticas para detectar diferencias significativas. Sin embargo, a pesar de esta variabilidad, a nivel de especies se detectó una 
(E)

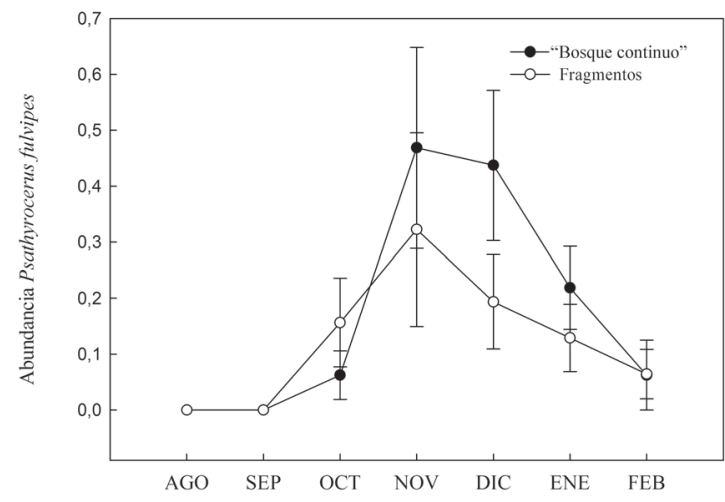

(G)

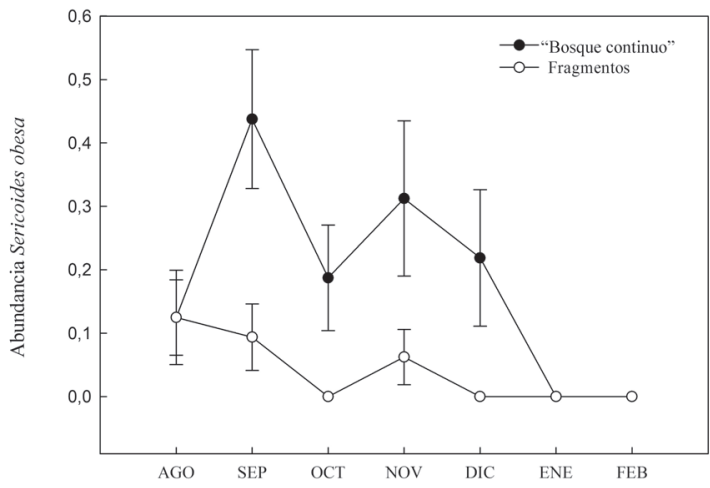

(F)

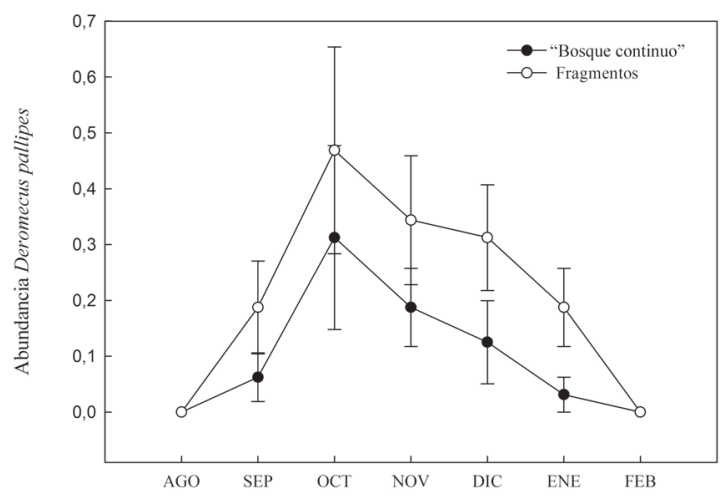

(H)

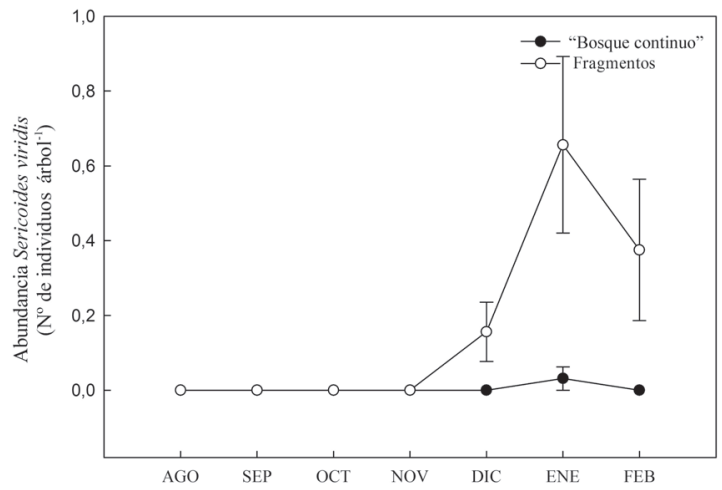

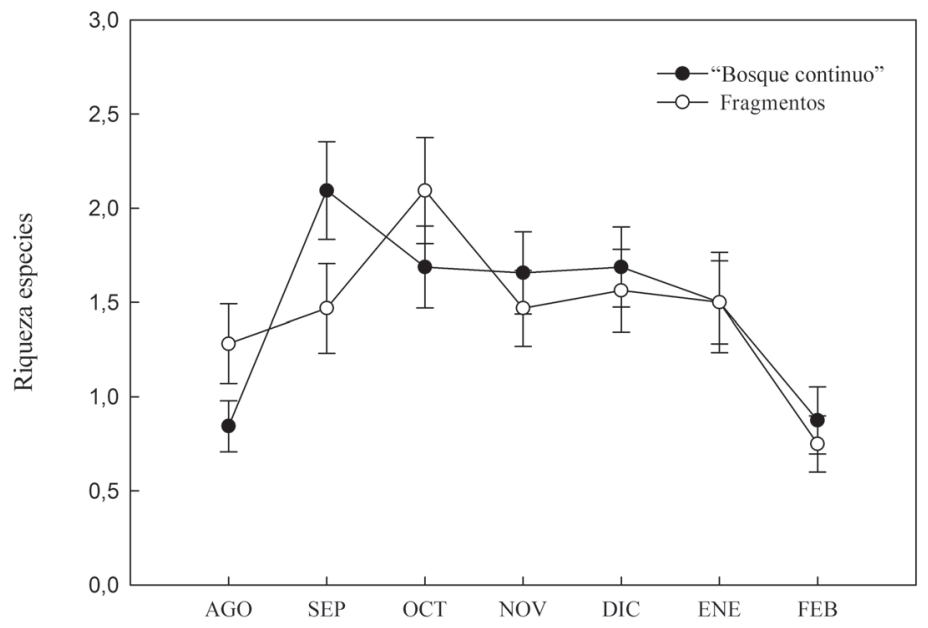

Fig. 2: Riqueza de especies de insectos defoliadores asociados a A. chilensis (promedio por árbol \pm $1 \mathrm{EE}$ ) en el bosque continuo y fragmentos de bosque maulino, entre agosto 2005 y febrero 2006.

Species richness of folivorous insects associated with A. chilensis (mean per tree $\pm 1 \mathrm{SE}$ ) in the continuous forest and small fragments of Maulino forest, between August 2005 and February 2006. 
respuesta diferencial, siendo algunas más abundantes en el bosque continuo y otras en los fragmentos. Incluso, dentro del mismo género, algunas especies respondieron de manera diferente a la fragmentación del bosque maulino. Por ejemplo, entre los Scarabaeidae, $S$. obesa fue más abundante en el bosque continuo en tanto $S$. viridis lo fue en los fragmentos; entre los Chrysomelidae, Psathyrocerus sp. 1 fue más abundante en el bosque continuo en tanto $P$. fulvipes obtuvo abundancias similares en ambos hábitat. Otras especies del mismo género como $P$. pallens y $P$. pyrroptera fueron más abundantes en los fragmentos de bosque, así como también el Elateridae $D$. pallipes.

La riqueza de especies por árbol tampoco fue afectada por la fragmentación del bosque maulino, aunque en total se encontraron más especies en el conjunto de fragmentos que en bosque continuo, con 63 y 46 especies, respectivamente. Sin embargo, la composición de especies de insectos sí cambió con la fragmentación del bosque. De las 77 especies capturadas durante el estudio, solo 30 fueron compartidas por el bosque continuo y los fragmentos, 32 fueron halladas solo en los fragmentos y 14 solo en el bosque continuo. Cuando se analizó la similitud de especies entre bloques al interior del bosque continuo, entre fragmentos y entre los bloques del bosque continuo y fragmentos, según el índice de similitud Sorensen, que incluye solo la presencia de especies, la fauna de insectos defoliadores fue parecida en todas las comparaciones, compartiéndose en promedio

\section{Encadenamiento promedio}

Distancia: (euclidiana)

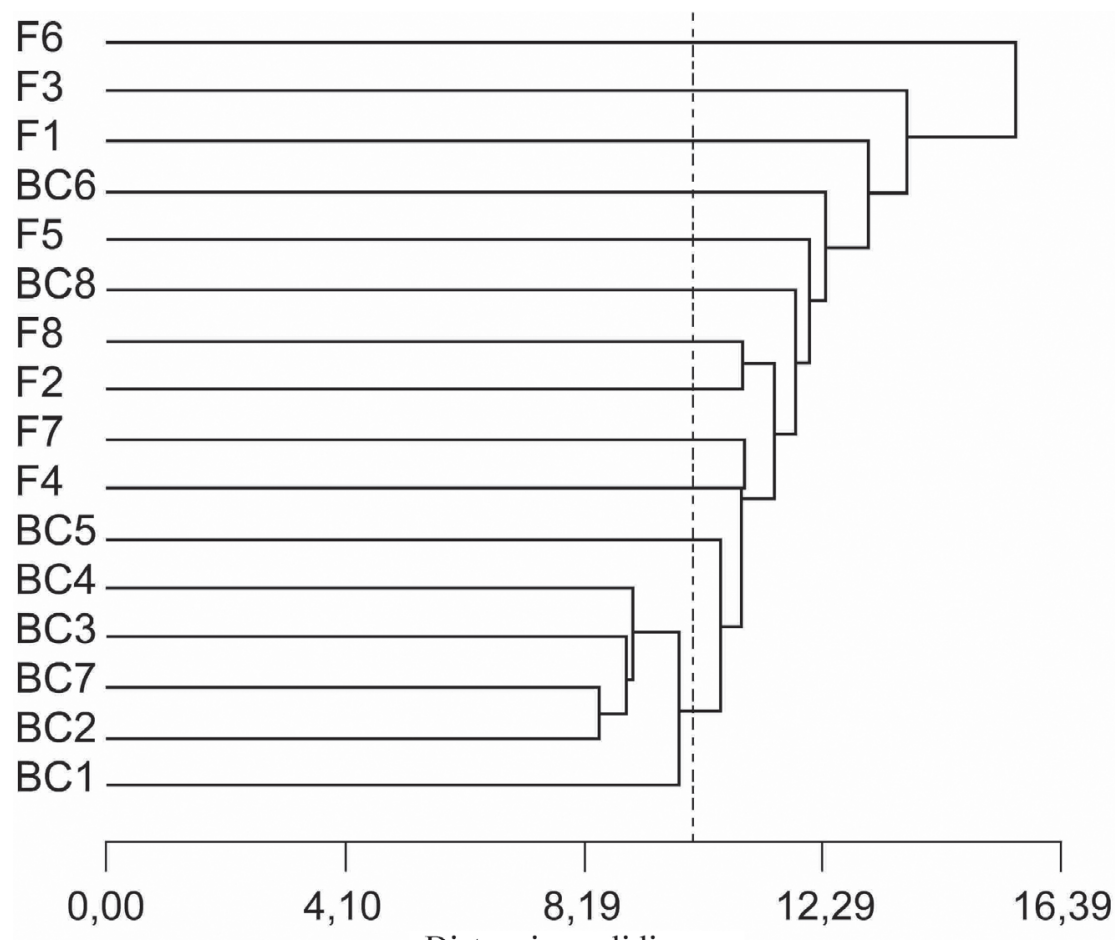

Distancia euclidiana

Fig. 3: Dendrograma de similitud, considerando la abundancia de las diferentes especies de insectos defoliadores asociados a A. chilensis en los 16 bloques (ocho del bosque continuo, BC, y ocho de los fragmentos, F). La línea punteada perpendicular al eje de similitud representa el valor crítico (para $\mathrm{a}=0,05)$ obtenido a partir de la distribución nula $(\mathrm{n}=100$ iteraciones).

Similarity dendrogram, considering the abundance of the different species of folivorous insects associated with A. chilensis in the 16 blocks (eight from the continuous forest, BC, and eight from small fragments, F). The perpendicular dotted line on the similarity axis represents the critical value (for $\mathrm{a}=0.05$ ) obtained from the null distribution $(\mathrm{n}=100$ iterations). 
cerca del $70 \%$ de las especies. En tanto según el índice de Morisita Horn, que incorpora además las abundancias relativas de las especies, la similitud de la fauna de insectos defoliadores en general disminuyó en relación al índice de Sorensen, sugiriendo que si bien se comparte un alto número de especies, sus abundancias relativas varían de un bloque a otro. Además, según este índice, la similitud entre bloques del bosque continuo fue superior $(0,17)$ que cuando estos se comparan con los fragmentos $(0,13)$ y que la similitud entre los fragmentos $(0,09)$. Al hacer el análisis de conglomerados se observa que los bloques BC6 y $\mathrm{BC} 8$ son muy disímiles al resto de los bloques del bosque continuo. Ello posiblemente se deba a la heterogeneidad de hábitat que es posible encontrar en el bosque continuo, con sitios más abiertos e iluminados (más parecidos a los fragmentos) que otros. En efecto, el bloque BC8 se encuentra en el borde del bosque continuo.

La menor similitud de especies entre los fragmentos de bosque es consistente con el mayor número total de especies capturadas en el conjunto de los fragmentos, en comparación con el bosque continuo, a pesar de que la diversidad por árbol no varió. Ello sugiere una mayor diversidad gamma (a nivel de paisaje) en los paisajes fragmentados. Esto se puede deber al arreglo espacial de los fragmentos pequeños de bosque nativo, los que al estar separados y cubrir un área geográfica mayor que el bosque continuo capturan una mayor heterogeneidad de hábitat en el paisaje, mecanismo propuesto por Tscharntke et al. (2002). Debido a que por motivos logísticos en el bosque continuo no fue posible muestrear árboles en un área similar a la abarcada por el conjunto de los fragmentos, no es posible descartar que la mayor diversidad gamma se deba al mayor espaciamiento (y heterogeneidad de hábitat) de los fragmentos, y no consecuencia de la fragmentación.

Los resultados obtenidos en este estudio, en conjunto con otros realizados en la misma zona (Grez et al. 2003, Bustamante-Sánchez et al. 2004, Jaña-Prado \& Grez 2004), así como los realizados en otros paisajes fragmentados, indican que los efectos de la fragmentación pueden ser variados. La riqueza o abundancia puede disminuir, mantenerse o incluso aumentar a medida que el tamaño del fragmento se reduce (Kruess \& Tscharntke
2000). Por ejemplo, en Alemania la riqueza de especies de mariposas se correlaciona positivamente con el tamaño del fragmento (Tscharntke et al. 2002); lo mismo ocurre con la riqueza y abundancia de un ensamble de coleópteros en el bosque fragmentado de Fray Jorge, Chile (Barbosa \& Marquet 2002), así como también en coleópteros carroñeros en bosques fragmentados de la Amazonia y de Nueva York (Klein 1989, Gibbs \& Stanton 2001). Otras evidencias, sin embargo, muestran el efecto contrario, con un aumento en la abundancia y riqueza de especies con la fragmentación. Tal es el caso de lo que ocurre en fragmentos de bosque en Hungría y Ucrania, donde el número total de especies de carábidos (Coleoptera) aumenta en fragmentos más pequeños (Magura et al. 2001). En agroecosistemas también se ha observado un incremento de la densidad poblacional y riqueza de especies de coleópteros en ambientes más fragmentados (Grez et al. 2004a, 2004b). Revisiones recientes sobre el efecto de la fragmentación del hábitat muestran que las especies responden de manera diferente y con distinta intensidad a la fragmentación del hábitat debido a sus diferentes estrategias de historia de vida (Didham 1997, Ewers \& Didham 2006). Especies de niveles tróficos superiores, especialistas de hábitat, especies con tamaños corporales grandes y con poca capacidad de dispersión son más susceptibles a la fragmentación del hábitat y a la extinción Ewers \& Didham 2006). Por ello, para comprender los efectos de la fragmentación del bosque maulino es necesario conocer la historia natural de las especies que habitan este bosque y su importancia en el funcionamiento ecosistémico (Jaña-Prado \& Grez 2004).

La baja abundancia de insectos defoliadores (con un promedio de solo 13,91 $\pm 0,87$ individuos por árbol) coincide con los bajos niveles de herbivoría (12\% de defoliación de tejido vegetal) registrados en el bosque maulino en adultos de A. chilensis (Vásquez et al. 2007) y en plántulas de Cryptocarya alba (Vega 2001). Además, el patrón de abundancia de los insectos herbívoros de más alta representación en el bosque maulino es coherente con el patrón de defoliación descrito para A. chilensis en este bosque. En las primeras cohortes de hojas, al principio de la temporada de expansión foliar (septiembre), la herbivoría es 
mayor en el bosque continuo que en los fragmentos (Vásquez et al. 2007). Al mismo tiempo, según los resultados de este estudio, la abundancia de $S$. obesa, insecto de gran tamaño (1 $\mathrm{cm}$ de largo aprox.) y que remueve grandes cantidades de superficie foliar, es significativamente mayor en el bosque continuo que en los fragmentos. Al avanzar en la temporada, las diferencias en herbivoría entre hábitat desaparecen, junto con el incremento de la abundancia en los fragmentos de insectos como los Chrysomelidae y $S$. viridis. Por ello es muy probable que estos insectos sean los principales responsables de los patrones de defoliación de A. chilensis en el bosque maulino. Mediciones posteriores a este estudio confirman estos patrones de herbivoría y abundancia de insectos herbívoros en el bosque continuo y fragmentos de bosque Maulino (X. De la Vega, resultados no publicados).

Todos los insectos defoliadores encontrados tanto en el bosque continuo como en los fragmentos corresponden a especies nativas. Lo mismo se observó en la fauna de coleópteros asociados a la descomposición de heces de ganado bovino (Bustamante-Sánchez et al. 2004), como también en los insectos herbívoros asociados al follaje de cuatro especies arbóreas (Jaña-Prado \& Grez 2004). En otro trabajo realizado en la zona, solamente dos especies de coleópteros epigeos introducidos, asociados a coníferas exóticas, fueron recolectadas en las plantaciones de pino en muy baja abundancia y nunca fueron capturadas en los fragmentos del bosque maulino (Grez et al. 2003). La dominancia de especies nativas y la rara ocurrencia de especies de insectos exóticos sugiere que, a pesar de la fragmentación del bosque maulino y de la gran extensión ocupada por las plantaciones de pino en la zona, no ha ocurrido una llegada masiva y establecimiento de especies invasoras y que la entomofauna nativa estaría relativamente bien conservada. El tipo de matriz que rodea a los remanentes del bosque maulino sin duda ha ayudado a mantener la fauna. Las plantaciones de pino que están en la zona de la Reserva Nacional Los Queules tienen un manejo que permite el establecimiento de un abundante sotobosque mayoritariamente compuesto por especies nativas, incluida $A$. chilensis, la que alcanza altas densidades allí. Desde el punto de vista de esta especie, el paisaje puede ser considerado un continuo. Sin embargo, a pesar de esta continuidad en la distribución del maqui, algunos insectos defoliadores cambian su abundancia en el paisaje. Esto podría deberse a cambios en las condiciones abióticas y bióticas producto de la fragmentación, que afectan al maqui y/o a los insectos defoliadores. Así por ejemplo, se sabe que con la fragmentación del bosque maulino, incrementa la luminosidad y decrece la humedad del suelo (Henríquez 2002, Burgos et al. 2008), incrementa la densidad de individuos (Lagos 2007), el contenido de nitrógeno y el grosor de las hojas, y disminuye el área foliar de A. chilensis (Repetto et al. 2007), incrementa la abundancia de aves insectívoras y la insectivoría (González-Gómez et al. 2006), todos factores que podrían ser relevantes para las poblaciones de insectos defoliadores asociados a A. chilensis. Además, con la fragmentación de este bosque cambian las abundancias relativas de otras especies arbóreas (Bustamante et al. 2005), lo que también podría ser relevante para estos insectos, considerando que no necesariamente serían especialistas del maqui.

En general, la fragmentación y pérdida del hábitat son consideradas las mayores amenazas para la biodiversidad (Myers 1988, Groom \& Schumaker 1993, FAO 2002). Estas perturbaciones son cada vez más frecuentes y muchas veces irreversibles, si no se toman medidas para restaurar el paisaje. Por ello, los fragmentos pequeños de bosque son una realidad. En este estudio mostramos que la fauna de insectos defoliadores asociados a los fragmentos remanentes del bosque maulino no sufre grandes cambios respecto al bosque continuo. Además, el 41,5\% de las especies de insectos defoliadores está solo presente en los fragmentos, e incluso, el conjunto de fragmentos soporta una mayor diversidad de especies nativas que el bosque continuo. Otros grupos de insectos nativos, aves, roedores también desarrollan abundantes poblaciones en estos fragmentos. Esto sugiere que los fragmentos del bosque maulino, a pesar de su tamaño o aislamiento, son un importante reservorio de biodiversidad nativa y deben ser conservados (Grez 2005). Ellos son de propiedad privada, lo que hace necesario un plan de manejo público-privado para asegurar la conservación de este bosque (Simonetti et al. 2002, Simonetti 2006). 


\section{AGRADECIMIENTOS}

Agradecemos a Yuri Zúñiga por su ayuda en terreno, a Mario Elgueta (Museo Nacional de Historia Natural), Danilo Cepeda (Museo Entomológico Luis Peña de la Facultad de Ciencias Agronómicas de la Universidad de Chile) y Viviane Jerez (Universidad de Concepción) en la ayuda prestada en la identificación de los insectos. Agradecemos también a Masisa S.A. y CONAF VII Región por permitirnos trabajar en sus terrenos. Este trabajo fue financiado por FONDECYT 1050745.

\section{LITERATURA CITADA}

ARNOLD AE \& NM ASQUITH (2002) Herbivory in a fragmented tropical forest: patterns from islands at Lago Gatun, Panama. Biodiversity and Conservation 11: 1663-1680.

ARTIGAS JN (1994) Entomología económica. Insectos de interés agrícola, forestal, médico y veterinario (nativos, introducidos y susceptibles de ser introducidos). Editorial Aníbal Pinto, Concepción, Chile. 943 pp

BARBOSA O \& PA MARQUET (2002) Effects of forest fragmentation on the beetle assemblage at the relict forest of Fray Jorge, Chile. Oecologia 132: 296306.

BENÍTEZ-MALVIDO J, G GARCÍA-GUZMÁN \& ID KOSSMANN-FERRAZ (1999) Leaf-fungal incidence and herbivory on tree seedlings in tropical rainforest fragments: an experimental study. Biological Conservation 91: 143-150.

BENÍTEZ-MALVIDO J (2001) Regeneration in tropical rainforest fragments. En: Bierregaard Jr. RO, C Gascon, TE Lovejoy \& R Mesquita (eds) Lessons from Amazonia: the ecology and conservation of a fragmented forest: 136-145. Yale University Press, New Haven, Connecticut, USA.

BRESCIANO D, JA SIMONETTI \& AA GREZ (1999) Edge effects in a Mediterranean woodland of central Chile. Journal of Mediterranean Ecology 1: $35-40$.

BURGOS A, AA GREZ \& RO BUSTAMANTE (2008) Seed production, pre-dispersal seed predation and germination of Nothofagus glauca (Nothofagaceae) in a temperate fragmented forest in Chile. Forest Ecology and Management 255: 1226-1233.

BUSTAMANTE RO \& C CASTOR (1998) The decline of an endangered temperate ecosystem: the ruil (Nothofagus alessandrii) forest in central Chile. Biodiversity and Conservation 7: 1607-1626.

BUSTAMANTE RO, JA SIMONETTI, AA GREZ \& J SAN MARTÍN (2005) La fragmentación del bosque maulino y su dinámica regeneracional: diagnóstico actual y perspectivas futuras, pp. 555-564. En: Smith C, JJ Armesto \& C Valdovinos (eds) Historia, biodiversidad y ecología de los bosques de la cordillera de la Costa. Editorial Universitaria, Santiago, Chile.

BUSTAMANTE RO, AA GREZ \& JA SIMONETTI (2006) Efectos de la fragmentación del bosque maulino sobre la abundancia y diversidad de especies nativas. En: Grez AA, JA Simonetti \& RO Bustamante (eds) Biodiversidad en ambientes fragmentados de Chile: patrones y procesos a diferentes escalas: 83-97. Editorial Universitaria, Santiago, Chile.

BUSTAMANTE-SÁNCHEZ MA, AA GREZ \& JA SIMONETTI (2004) Dung decomposition and associated beetles in a fragmented temperate forest. Revista Chilena de Historia Natural 77: 107-120.

CRAWLEY MJ (1985) Reduction of oak fecundity by lowdensity herbivore populations. Nature 314: 163164.

DIDHAM RK (1997) An overview of invertebrate responses to forest fragmentation. En: Watt AD, NE Stork \& MD Hunter (eds) Forests and insects: 303320. Chapman and Hall, London, United.Kingdom.

DONOSO DS, AA GREZ \& JA SIMONETTI (2004) Effect of forest fragmentation on the granivory of differently-sized seeds. Biological Conservation 115: 63-70

ECHEVERRÍA C, D COOMES, J SALAS, JM REYBENAYAS, A LARA \& A NEWTON (2006) Rapid deforestation and fragmentation of Chilean temperate forests. Biological Conservation 130: 481-494.

EWERS R \& R DIDHAM (2006) Confounding factors in the detection of species responses to habitat fragmentation. Biological Reviews 81: 117-142.

FAO (FOOD AND AGRICULTURE ORGANIZATION OF THE UNITED NATIONS) (2002) Evaluación de los recursos forestales mundiales 2000. Roma, Italia. $367 \mathrm{pp}$.

FORTIN MJ \& J GUREVITCH (1993) Mantel tests: spatial structure in field experiments. En: Scheiner SM \& J Gurevitch (eds) Design and analysis of ecological experiments: 342-359. Chapman \& Hall, New York, USA.

FUENTES ER, J ETCHEGARAY, ME ALJARO \& G MONTENEGRO (1981) Shrub defoliation by matorral insects. En: Di Castri F, DW Goodlall \& RL Specht (eds) Mediterranean-type shrublands: 345-359. Elsevier, Amsterdam, Holland.

GIBBS JP \& EJ STANTON (2001) Habitat fragmentation and arthropod community change: carrion beetles, phoretic mites and flies. Ecological Applications 11: 79-85.

GONZÁLEZ-GÓMEZ P, CF ESTADES \& JA SIMONETTI (2006) Strengthened insectivory in a temperate fragmented forest. Oecologia 148: 137143.

GREZ AA (2005) El valor de los fragmentos pequeños del bosque maulino en la conservación de la fauna de coleópteros epigeos. En: Smith-Ramírez C, J Armesto \& C Valdovinos (eds) Biodiversidad y ecología de los bosques de la cordillera de la Costa de Chile: 565-572. Editorial Universitaria, Santiago, Chile.

GREZ AA, RO BUSTAMANTE, JA SIMONETTI \& L FAHRIG (1997) Landscape ecology, deforestation and habitat fragmentation: the case of the ruil forest in Chile. En: Salinas-Chávez E \& J Middleton. (eds) Landscape ecology as a tool for sustainable development in Latin America. Disponible en: www.brocku.ca/epi/lebk/lebk.html. [consulta: 15/ 05/2007]

GREZ AA, P MORENO \& M ELGUETA (2003) Coleópteros (Insecta, Coleoptera) epigeos asociados al bosque maulino y plantaciones de pino aledañas. Revista Chilena de Entomología 29: 9-18. 
GREZ AA, T ZAVIEZO \& S REYES (2004a) Short-term effects of habitat fragmentation on the abundance and species richness of beetles in experimental alfalfa micro-landscapes. Revista Chilena de Historia Natural 77: 547-558.

GREZ AA, T ZAVIEZO, L TISCHENDORF \& L FAHRIG (2004b) A transient, positive effect of habitat fragmentation on insect population densities. Oecologia 141: 444-451.

GROOM MJ \& N SCHUMAKER (1993) Evaluating landscape change: pattern of worldwide deforestation and local fragmentation. En: Kareiva PM, JG Kingsolver \& RB Huey (eds) Biotic interactions and global change: 24-44. Sinauer, Sunderland, Massachusetts, USA.

HENRÍQUEZ C (2002) El dilema de Lapageria rosea en bosques fragmentados: ¿cantidad o calidad de la progenie? Tesis Doctoral, Facultad de Ciencias, Universidad de Chile, Chile. 133 pp.

HOCHWENDER CG, VL SORK \& RJ MARQUIS (2003) Fitness consequences of herbivory on Quercus alba. American Midland Naturalist 150: 246-253.

INFOSTAT (2003) InfoStat versión 1.5. Grupo InfoStat, FCA, Universidad Nacional de Córdoba, Argentina.

JAÑA-PRADO R \& AA GREZ (2004) Insectos herbívoros en el bosque maulino: un ecosistema fragmentado. Revista Chilena de Entomología 30: 27-43

KLEIN BC (1989) Effects of forest fragmentation on dung and carrion beetle communities in central Amazonia. Ecology 70: 1715-1725.

KREBS CJ (1989) Ecological methodology. Harper and Row, New York, New York, USA. 654 pp.

KRUESS A \& T TSCHARNTKE (2000) Species richness and parasitism in a fragmented landscape: experiments and field studies with insects on Vicia sepium. Oecologia 122: 129-137.

LAGOS N (2007) Distribución de especies arbóreas en un bosque maulino fragmentado. Memoria de Título, Facultad de Ciencias Agronómicas, Universidad de Chile, Santiago, Chile. 53 pp.

MAGURA T, V KODOBOCZ \& B TÓTHMÉRÉSZ (2001) Effects of habitat fragmentation on carabids in forest patches. Journal of Biogeography 28: 129138.

MORENO CE (2001) Métodos para medir la biodiversidad. M\&T- Manuales y tesis SEA, Volumen 1. España. 84 pp.

MYERS N (1988) Tropical forest and their species. Going, going...? En: Wilson EO (ed) Biodiversity: 28-35. Academic Press, New York, New York, USA.

MYERS N, RA MITTERMEIER, CG MITTERMEIER, GAB DA FONSECA \& J KENT (2000) Biodiversity hotspots for conservation priorities. Nature 403: 853-858

NOSS RF (1990) Indicators for monitoring biodiversity: a hierarchical approach. Conservation Biology 4: 355-364.

POPTOOLS (2006) Versión 2.7.5, Australia: www.cse.csiro.au/poptools/

REPETTO-GIAVELLI F, LA CAVIERES \& JA SIMONETTI (2007) Respuestas foliares de
Aristotelia chilensis a la fragmentación del bosque maulino. Revista Chilena de Historia Natural 80: 469-477.

RICKLEFS R \& G MILLER (1999) Ecology. Fourth edition. Freeman and Company, New York, New York, USA. $481 \mathrm{pp}$.

ROLAND J (1993) Large-scale forest fragmentation increases the duration of tent caterpillar outbreak. Oecologia 93: 25-30.

SAAVEDRA B \& JA SIMONETTI (2001) New records of Dromiciops gliroides (Marsupialia: Microbiotheriidae) and Geoxus valdivianus (Rodentia: Muridae) in central Chile: their implications for biogeography and conservation. Mammalia 65: 96-100.

SAN MARTÍN J \& C DONOSO (1996) Estructura florística e impacto antrópico en el bosque maulino y plantaciones de pino aledañas. En: Armesto J, C Villagrán \& M Arroyo (eds) Ecología de los bosques nativos de Chile: 153-168. Editorial Universitaria, Santiago, Chile

SIMONETTI JA (2006) Conservación de biodiversidad en ambientes fragmentados: el caso del bosque maulino. En: Grez AA, JA Simonetti \& RO Bustamante (eds) Biodiversidad en ambientes fragmentados de Chile: patrones y procesos a diferentes escalas: 215-229. Editorial Universitaria, Santiago, Chile.

SIMONETTI JA, AA GREZ \& RO BUSTAMANTE (2002) El valor de la matriz en la conservación ambiental. Ambiente y Desarrollo (Chile) 18: 116118.

SIMONETTI JA, AA GREZ \& RO BUSTAMANTE (2006) Interacciones y procesos en el bosque maulino fragmentado. En: Grez AA, Simonetti JA \& RO Bustamante (eds). Biodiversidad en ambientes fragmentados de Chile: patrones y procesos a diferentes escalas: 99-114. Editorial Universitaria, Santiago, Chile.

SIMONETTI JA, AA GREZ, JL CELIS-DIEZ \& RO BUSTAMANTE (2007) Herbivory and seedling performance in a fragmented temperate forest of Chile. Acta Oecologica 32: 312-318.

STATSOFT (2001) Statistica data analysis software system, version 6., Statsoft. Inc., Tulsa, Oklahoma, USA; <www.statsoft.com> [consulta: 08/11/2006].

TSCHARNTKE T, I STEFFAN-DEWENTER, A KRUESS \& C THIES (2002) Contribution of small habitat fragments to conservation of insects communities of grassland-cropland landscapes. Ecological Applications 12: 354-363.

VÁSQUEZ PA, AA GREZ, RO BUSTAMANTE \& JA SIMONETTI (2007) Herbivory, foliar survival and shoot growth in fragmented populations of Aristotelia chilensis. Acta Oecologica 31: 48-53.

VEGA MI (2001) Efecto de la fragmentación del bosque maulino sobre la herbivoría en plántulas de Cryptocarya alba. Memoria de Título, Medicina Veterinaria, Facultad de Ciencias Veterinarias y Pecuarias, Universidad de Chile, Santiago, Chile. $79 \mathrm{pp}$. 